\title{
Evaluation of Midpalatal Suture Ossification Using Cone-Beam Computed Tomography: A Digital Radiographic Study
}

\author{
Girish Katti ${ }^{1}$, Syed Shahbaz ${ }^{1, *}$, Chandrika Katti², Mohd Sabyasachi Rahman ${ }^{1}$
}

\begin{abstract}
Background: Cone beam computed tomography (CBCT) imaging techniques are the recent rage in the field of oral diagnostic imaging modality. It is noninvasive, faster and lacks anatomic superimposition. Earlier maxillary occlusal radiographs were used to assess and evaluate the mid palatal suture, but being a two dimensional imaging modality it could not assess the ossification process which takes place in multiple planes mostly due to curved nature of the palate. In this study we assessed the mid palatal suture morphology and classify them according to the variants using CBCT images.

Materials and methods: A total of 200 CBCT scans (95 males and 105 females) were evaluated in the present study from the archives of an imaging center. As per Angelieri classification the midpalatal suture was classified into five categories (A-E) depending on the degree of ossification that had taken place. Statistical analysis was done by Chi Square test using SPSS version 23.0.

Results: There is statistically significant difference present in the stages of maturity of mid palatal suture in various age groups with Stage B is most common in Group 1 (50\%), Stage C most common in Group 2 (60\%) and Group 3 (40\%) and Stage E more common in Group 4 (50\%). Conclusion: The results of the present study showed a wide variation in the initiation time and the degree of ossification and morphology of the midpalatal suture in different age groups. Although there was an increase in the closure of the suture with aging, age is not a reliable criterion for determining the open or closed nature of the suture. This finding is important in providing an idea as to how diverse is the ossification of maxillary sutures.
\end{abstract}

KEYWORDS

$\mathrm{CBCT}$; midpalatal suture; ossification; maturation; maxillary suture

\section{AUTHOR AFFILIATIONS}

${ }^{1}$ Department of Oral Medicine and Radiology, Al-Badar Dental College and Hospital, Kalaburgi, Karnataka, India

2 Department of Orthodontia, Al-Badar Dental College and Hospital, Kalaburgi, Karnataka, India

* Corresponding author: Department of Oral Medicine and Radiology, Al-Badar Dental College and Hospital, Naganhalli Road, Gulbarga-585102, Karnataka, India; e-mail: drsshahbaz@gmail.com 


\section{INTRODUCTION}

A pristine and aesthetically beautiful smile is important to many, and so to get their smiles as beautiful as possible more and more people are opting for orthodontic corrections. Moreover, it is not just for aesthetics that orthodontic treatments are done, the more the awareness penetrates deep into the society, the more people understand that proper alignment of teeth not only helps in aesthetics but also helps in optimum function and boosts confidence and improves the quality of life of a person. For proper orthodontic planning, CBCT plays a significant role. CBCT is the recent rage in craniofacial imaging modality which provides for accurate three-dimensional images without any overlapping.

Unlike other cranial sutures in our body, the mid palatal suture is the solitary suture that might not close up in the elderly. This midpalatal suture plays a vital role in a certain orthodontic procedure like Rapid Maxillary Expansion (RME) which helps in correcting transverse discrepancies of the maxilla by applying force with in-office appliances to expand the maxillary width $(1,2)$. However, with the fusion of the maxillary suture, the procedure of correcting the transverse discrepancy cannot be done only with appliances and needs to be surgically assisted thus giving rise to a procedure known as Surgically Assisted Rapid Maxillary Expansion (SARME) (3). Several studies on the morphology, initiation time and degree of fusion of the suture in different individuals have yielded wide variations concerning initiation time and progression of suture closure; a few cases over 18 years of age have reported completely open sutures, and hence the decision whether to do an RME or SARME cannot be based only on the chronological age and needs to be assessed as per individual patients $(4,5)$.

The use of maxillary occlusal radiographs did earlier the assessment of the midpalatal suture, but this method was not without flaws. Since the occlusal radiographs are a $2 \mathrm{D}$ representation of a $3 \mathrm{D}$ structure and so due to the superimposition of the nasal structures and the vomer bone on the midpalatal area, misinterpretation of the radiograph and discrepancy with morphologic findings are possible. This is where the CBCT comes in as the latest offering in the field of dental imagery solving the problems faced with the $2 \mathrm{D}$ imaging techniques. CBCT provides high-resolution 3D images of craniofacial structures $(6,7)$. Added advantages are high dimensional accuracy, noninvasive nature, time-saving, easy access, and lack of any anatomic superimpositions. In light of the above literature, given the discrepancies amongst the results of studies regarding the initiation time and maturation of the midpalatal suture (8-10), this study was done to evaluate the midpalatal suture morphology in various age groups.

\section{MATERIALS AND METHODS}

CBCT is easily accessible these days' orthodontists are not leaving anything to chance and are referring for CBCT scans when in doubt and are asking for an opinion regarding the radio analysis of mid palatal suture ossification and maturation. The sample of this particular study has been taken from one such scan center where patients are referred for CBCT analysis.

A total of 200 full volume CBCT scans showing the entire maxilla using the NewTom ${ }^{\circledR}$ Giano CBCT machine with the NNT software were collected from the archival records of the CBCT scan centre. The ethical clearance to conduct the study was obtained from the Institutional Ethical Committee. Care was taken not to include any scans of subjects who were undergoing orthodontic treatments or those having any pathologies in the maxilla. Out of the 200 scans, 95 were of male and 105 were of female

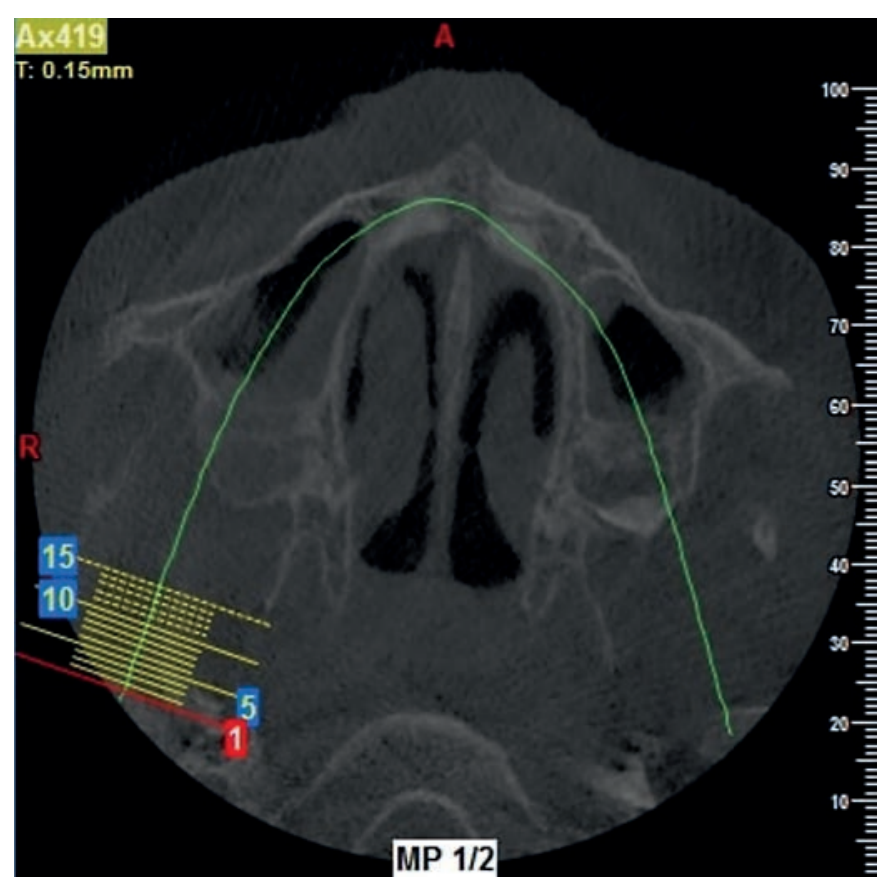

Fig. 1 Stage $A$, the mid palatal suture is almost a straight highdensity sutural line with no or little inter digitations.

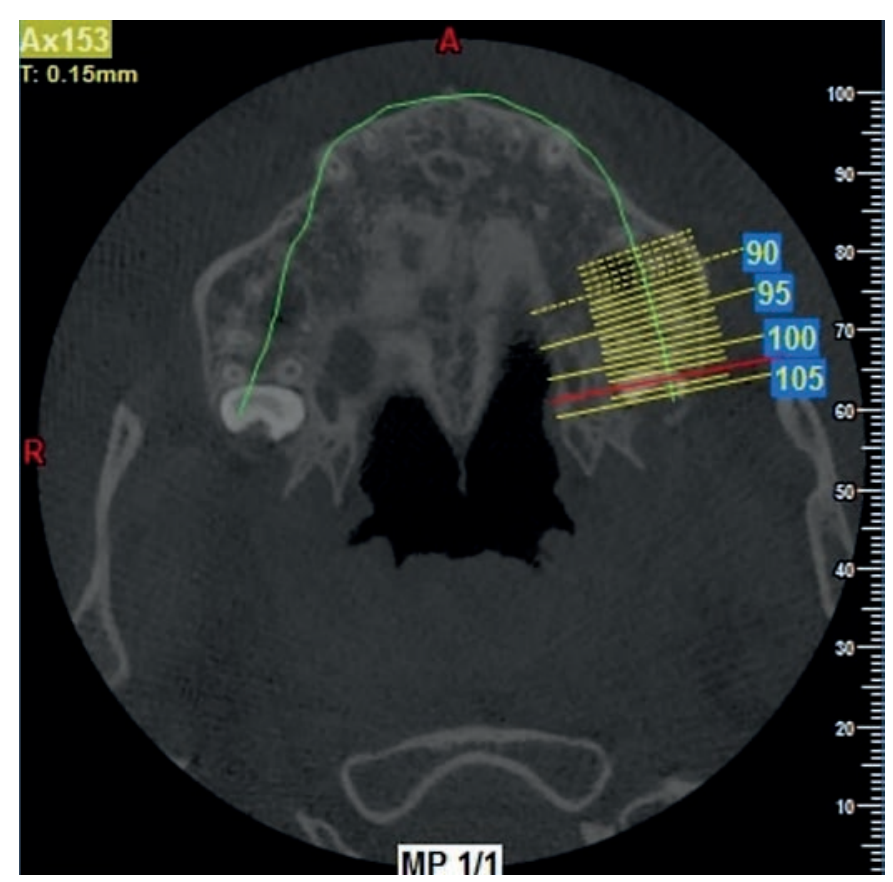

Fig. 2 Stage $B$, the mid palatal suture assumes an irregular shape and appears as a scalloped high density line. 


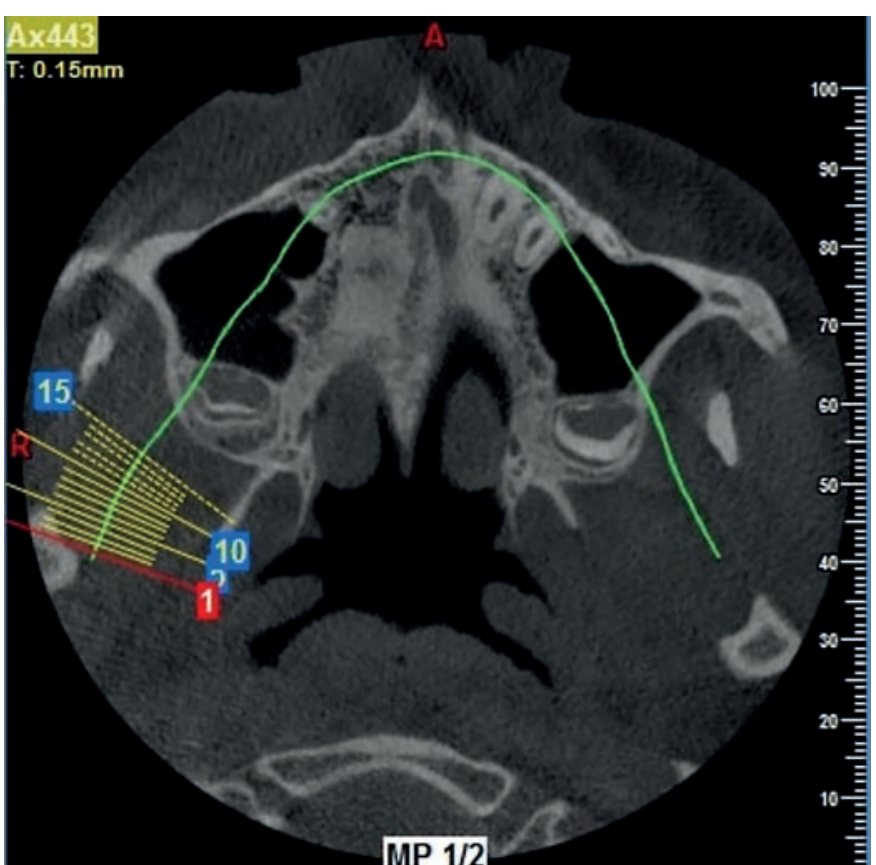

Fig. 3 Stage C, the mid palatal suture appears as 2 parallel, scalloped, high-density lines that are close to each other, separated by small low-density spaces in the maxillary and palatine bones.

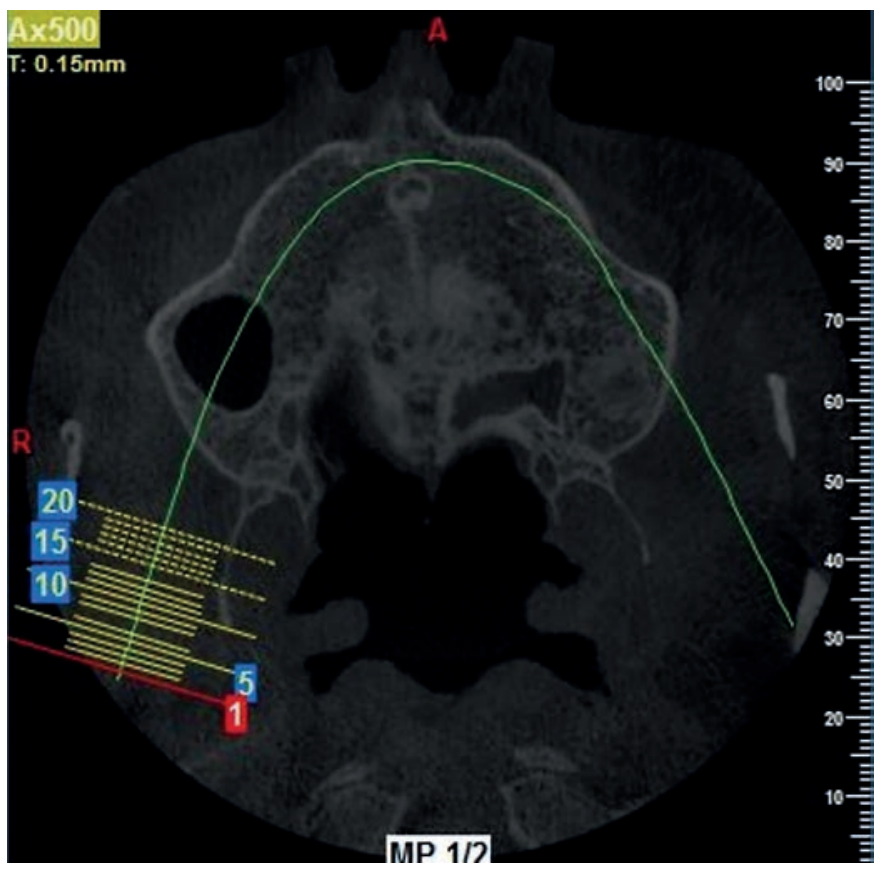

Fig. 4 Stage $D$, the fusion of the mid palatal suture has occurred in the palatine bone, with maturation progressing from posterior to anterior. In the palatine bone, the mid palatal suture cannot be visualized at this stage.

patients with age ranging from 11 years to 50 years. The precaution was taken to not in any way disclose the identity of the scans. The scans were obtained through a period of 12 months from November 2018 to October 2019. The scans were then grouped into 10-year age groups, like 11-20years, 21-30years, 31-40years, and 41-50years. These groups were named as group 1, group 2, group 3 and group 4 , respectively.

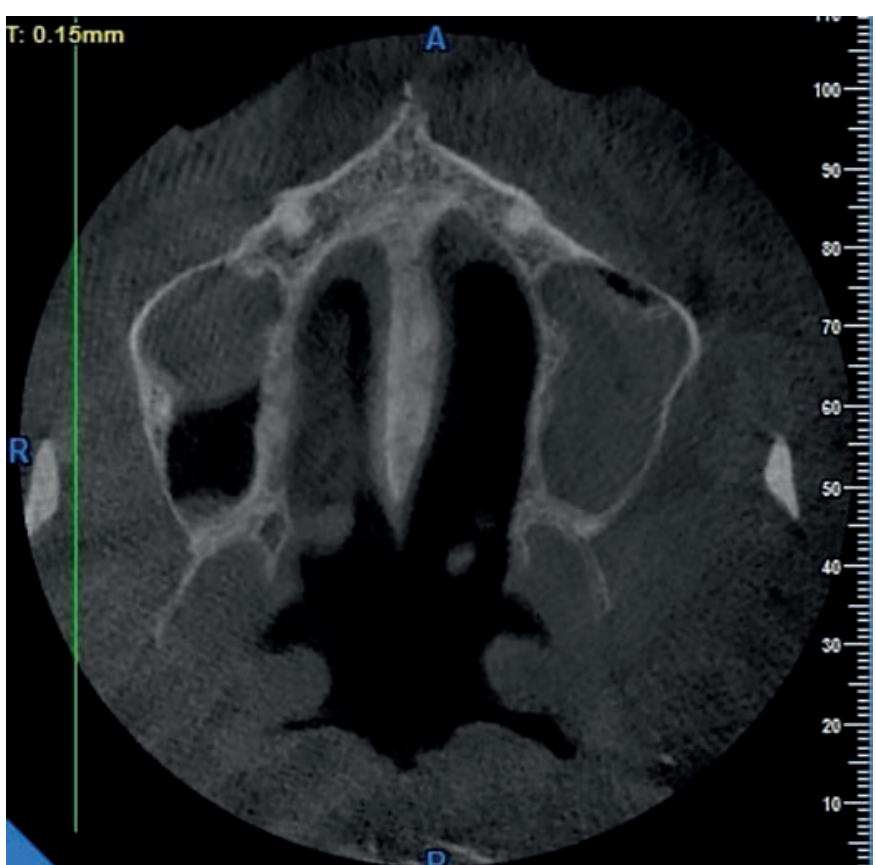

Fig. 5 Stage $E$, fusion of the mid palatal suture has occurred in the maxilla. The actual suture is not visible in at least a portion of the maxilla.

The classification of the morphology of the midpalatal suture was done based on the classification done by Angilieri et al. in their study (9). The classification is as follows:

In stage $A$, the mid palatal suture is almost a straight high-density sutural line with no or little interdigitations (Fig. 1).

In stage $B$, the midpalatal suture assumes an irregular shape and appears as a scalloped high-density line (Fig. 2).

In stage $\mathrm{C}$, the midpalatal suture appears as 2 parallel, scalloped, high-density lines that are close to each other, separated by small low-density spaces in the maxillary and palatine bones (between the incisive foramen and the Palatino-maxillary suture and posterior to the Palatino-maxillary suture) (Fig. 3).

In stage $\mathrm{D}$, the fusion of the midpalatal suture has occurred in the palatine bone, with maturation progressing from posterior to anterior. In the palatine bone, the midpalatal suture cannot be visualized at this stage, and the para-sutural bone density is increased (high-density bone) compared with the density of the maxillary para-sutural bone. In the maxillary portion of the suture, fusion has not yet occurred, and the suture still can be seen as 2 high-density lines separated by small low-density spaces (Fig. 4).

In stage $\mathrm{E}$, the fusion of the midpalatal suture has occurred in the maxilla. The actual suture is not visible in at least a portion of the maxilla. The bone density is the same as in other regions of the palate (Fig. 5).

The scans were assessed in a well-lit room by a single observer, twice after a gap of 5 days to do away with any intra-observer ambiguity. A weighted kappa coefficient was calculated to evaluate the intraexaminer agreement for the classification of the midpalatal suture maturation stages. The results of the intraclass correlation coefficient (ICC) test revealed high reliability between the two 


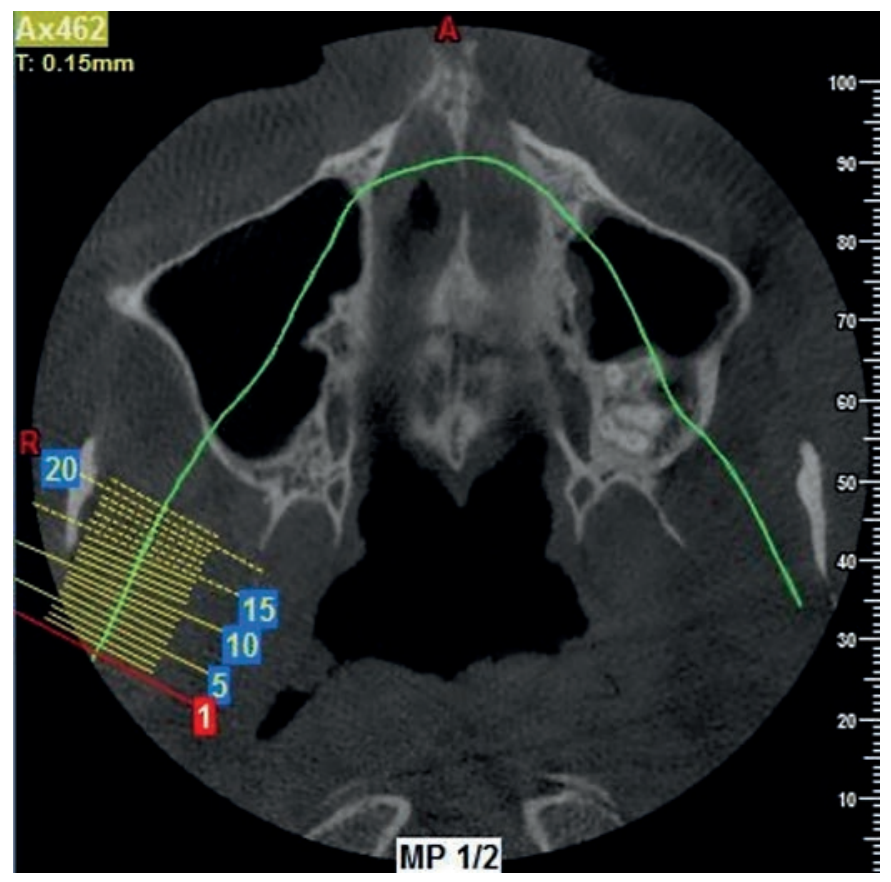

Fig. 6 Stage D', modification of Stage D.

assessments for all regions (ICC > 0.8). Thus the scans were classified into Stages A, B, C, D or E and were grouped as per the age groups. It was then analyzed by the Chi-Square test to find out if any significant differences are there amongst the groups. The statistical analysis was done using SPSS version 23.0, Chicago, USA.

\section{RESULTS}

The observer analyzed the 200 scans, they were classified under the 4 age groups, which were the groups 1-4, and the scans were distributed among the age groups as per the Angelieri classification. After analyzing twice by the observer, there were no statistically significant changes in both the readings.
A total of 15 scans were in stage A, 40 were in stage B, 70 scans were classified as stage $C, 25$ were in stage $D$ and 40 in stage E. Since A, B and C were the groups in which there is no fusion of the mid palatal suture it can be said that over $50 \%$ of the samples had no fusion in the midpalatal suture.

Apart from the 5 morphological classifications mentioned by Angelieri et al. (9), we found that there was one more variant of midpalatal suture maturation where the ossification process was not continuous but in bouts along the suture line. We named this as stage D' which was a modification of $\mathrm{D}$, and 10 samples were there in this class (Fig. 6). A noteworthy thing in the results is that about 75 scans of subjects over 20 years of age were either in class A, B or C. that means $50 \%$ of the samples over 20 years of age had open sutures (Table 1 and 2).

There was a statistically significant difference in the stages of maturity of the mid palatal suture in various age groups with Stage B (50\%) is most common in Group 1, stage $C$ most common in Group $2(60 \%)$ and Group $3(40 \%)$ and Stage $\mathrm{E}$ more common in Group 4 (50\%).

The results showed a gradual maturation in ossification of the midpalatal suture with an increase in age, but a specific age could not be determined in which the suture fuses completely. Furthermore, no significant finding could be assessed regarding the maturation pace concerning gender.

\section{DISCUSSION}

The increasing awareness of oral health and smile appeal is making people more concerned with proper aesthetics and overall health of the oral cavity. This is one of the main reasons for the ever-increasing cases at the orthodontists. The dental treatment being individual-specific as always, the midpalatal suture ossification of an individual carries an important role in orthodontic treatment if there is a discrepancy in the palatal width. Since after many previous studies, no particular age could be confirmed for the fusion of the midpalatal suture this particular study was

Tab. 1 Mean and SD of age in various age groups according to gender.

\begin{tabular}{|c|c|c|c|c|c|c|}
\hline Age Group & Gender & $\mathbf{N}$ & Minimum & Maximum & Mean & Std. Deviation \\
\hline \multirow[t]{3}{*}{$11-20$ years } & Female & 35 & 11.0 & 17.0 & 14.71 & 1.98 \\
\hline & Male & 15 & 13.0 & 18.0 & 15.33 & 2.52 \\
\hline & Total & 50 & 11.0 & 18.0 & 14.90 & 2.02 \\
\hline \multirow[t]{3}{*}{$21-30$ yeas } & Female & 20 & 21.0 & 30.0 & 23.50 & 4.36 \\
\hline & Male & 30 & 22.0 & 30.0 & 25.67 & 2.88 \\
\hline & Total & 50 & 21.0 & 30.0 & 24.80 & 3.49 \\
\hline \multirow[t]{3}{*}{$31-40$ years } & Female & 25 & 31.0 & 40.0 & 35.40 & 4.28 \\
\hline & Male & 25 & 31.0 & 40.0 & 36.00 & 4.58 \\
\hline & Total & 50 & 31.0 & 40.0 & 35.70 & 4.19 \\
\hline \multirow[t]{3}{*}{$41-50$ years } & Female & 25 & 45.0 & 50.0 & 46.80 & 2.17 \\
\hline & Male & 25 & 41.0 & 50.0 & 45.20 & 3.83 \\
\hline & Total & 50 & 41.0 & 50.0 & 46.00 & 3.06 \\
\hline
\end{tabular}


Tab. 2 Comparison of stages of mid palatal suture maturation.

\begin{tabular}{|c|c|c|c|c|c|}
\hline \multirow[t]{2}{*}{ Stage } & \multicolumn{4}{|l|}{ Group } & \multirow[t]{2}{*}{ Total } \\
\hline & 1 & 2 & 3 & 4 & \\
\hline A & $15(30 \%)$ & 0 & 0 & 0 & $5(7.5 \%)$ \\
\hline B & $25(50 \%)$ & $5(10 \%)$ & $5(10 \%)$ & $5(10 \%)$ & $40(20 \%)$ \\
\hline C & $10(20 \%)$ & $30(60 \%)$ & $20(40 \%)$ & $10(20 \%)$ & $70(35 \%)$ \\
\hline D & 0 & $5(10 \%)$ & $15(30 \%)$ & $5(10 \%)$ & $25(12.5 \%)$ \\
\hline E & 0 & $5(10 \%)$ & $10(20 \%)$ & $25(50 \%)$ & $40(20 \%)$ \\
\hline$X\left[D^{\prime}\right]$ & 0 & $5(10 \%)$ & 0 & $5(10 \%)$ & $10(5 \%)$ \\
\hline Total & $50(100 \%)$ & $50(100 \%)$ & $50(100 \%)$ & $50(100 \%)$ & $200(100 \%)$ \\
\hline Chi Sq & \multicolumn{2}{|l|}{154.714} & P value & \multicolumn{2}{|l|}{$0.000^{*}$} \\
\hline
\end{tabular}

* Statistically significant $(p<0.01)$

done to assess the ossification process on CBCT scans and classify as per there maturation progress. CBCT was chosen as it is better for any 2D imaging modality for its 3D analysis and majorly due to no structural overlap.

The results of the present study with the degree of ossification of the suture in the age groups 1-4, showed that ossification increased with ageing; however, some cases of completely open sutures were observed in people over 20year age groups.

A study done on patients over the age of 70 years revealed that the midpalatal suture is the only suture that might not close completely even in the elderly (11). Further, in another study subjects more than 40 years of age, showed a few cases of incomplete ossification of the suture, which was similar to the findings in our study (4). In one more study with the use of CBCT images, in only $13 \%$ of the adult subjects completely closed sutures were detected (12).

There was no significant relationship between the closure of the suture and age drawn from the results of 30 micro-CT analyses in a study carried on 28 patients, 14 to 17 years of age (8). Based on a hypothesis by Cohen $M M$, there is no relationship between the termination of growth and closure of the suture, i.e., even if $95 \%$ of the growth of the maxilla ends at 7 years of age, the suture is not necessarily closed (13).

The decrease in functional forces of muscles with ageing, teeth loss and soft diet, contribute to the open suture in adults (14); thus, the relative course of development such as morphology and ossification process of the midpalatal suture contrary to other cranial sutures depends on the masticatory forces exerted on the maxillary bone during life.

In a study by Angelieri et al. on CBCT images of 140 patients, subjects over 11 years of age exhibited all the developmental stages for the midpalatal suture. The chronological age was an invalid criterion for determining the developmental stage of the midpalatal suture during growth (9). In the present study, too, evaluation of the morphology of the suture in 10-year age groups revealed wide variations in subjects over 20 years of age, with $50 \%$ of the subjects over 20 years of age in stages A, B, and C of the development of the suture; in these stages, completely open sutures are observed.
In a study by Fricke-Zechet et al. it was reported that different individuals have varied morphologic changes in suture which are not completely related to age (15). In the present study, the suture morphology did not reveal any significant differences between genders, which are consistent with the results of a study by Revelo et al. (16). The results of the present study showed the posteroanterior direction of the ossification of the suture. The results of the present study showed the posteroanterior direction of ossification of the suture, similar to the study done by Melsen et al. (17). However, with exceptions where there were interrupted bouts of ossification along the suture line which could be said as a modification of D. Even though there was an increase in the closure of the suture with ageing, nevertheless, age is not a consistent measure for determining the open or closed nature of the suture.

\section{CONCLUSION}

There is ample variation in the initiation time and the degree of ossification and morphology of the midpalatal suture in different age groups. Even though there was an increase in the closure of the suture with ageing, stillage is not a consistent criterion for determining the open or closed nature of the suture. This finding is important in preparing an appropriate orthodontic treatment plan, irrespective of the patient's age. Further, studies with a larger sample size will spread more light on the maturation process of midpalatal suture.

\section{REFERENCES}

1. Lione R, Ballanti F, Franchi L, Baccetti T, Cozza P. Treatment and posttreatment skeletal effects of rapid maxillary expansion studied with low-dose computed tomography in growing subjects. Am J Orthod Dentofacial Orthop 2008; 134(3): 389-92.

2. Baccetti T, Franchi L, Cameron CG, McNamara JA. Treatment timing for rapid maxillary expansion. Angle Orthod 2001; 71(5): 343-50.

3. Chrcanovic BR, Custódio ALN. Orthodontic or surgically assisted rapid maxillary expansion. Oral Maxillofac Surg 2009; 13(3): 123.

4. Mir KPB, Mir APB, Mir MPB, Haghanifar S. A unique functional craniofacial suture that may normally never ossify: A cone-beam computed tomography-based report of two cases. Indian J Dent 2016; 7(1): 48 .

5. Wehrbein H, Yildizhan F. The mid-palatal suture in young adults. A radiological-histological investigation. Eur J Orthod 2001; 23(2): 105-14. 
6. Machado GL. CBCT imaging - A boon to orthodontics. Saudi Dental J 2015; 27(1): 12-21.

7. Kamburoğlu K. Use of dentomaxillofacial cone beam computed tomography in dentistry. World J Radiol 2015; 7(6): 128.

8. Korbmacher H, Schilling A, Püschel K, Amling M, Kahl-Nieke B. Age-dependent three-dimensional microcomputed tomography analysis of the human midpalatal suture. J Orofac Orthop 2007; 68(5): 364-76.

9. Angelieri F, Cevidanes LH, Franchi L, Gonçalves JR, Benavides E, McNamara Jr JA. Midpalatal suture maturation: classification method for individual assessment before rapid maxillary expansion. Am J Orthod Dentofacial Orthop 2013; 144(5): 759-69.

10. Haghanifar S, Mahmoudi S, Foroughi R, Mir APB, Mesgarani A, Bijani A. Assessment of midpalatal suture ossification using cone-beam computed tomography. Electron Physician 2017; 9(3): 4035.

11. N'Guyen T, Ayral X, Vacher C. Radiographic and microscopic anatomy of the mid-palatal suture in the elderly. Surg Radiol Anat 2008; $30(1): 65-8$.
12. N'Guyen T, Gorse FC, Vacher C. Anatomical modifications of the mid palatal suture during ageing: a radiographic study. Surg Radiol Anat 2007; 29(3): 253-9.

13. Cohen Jr MM. Sutural biology and the correlates of craniosynostosis. Am J Med Gen 1993; 47(5): 581-616.

14. Curtis N, Jones M, Evans S, O'Higgins P, Fagan M. Cranial sutures work collectively to distribute strain throughout the reptile skull. J R Soc Interface 2013; 10(86): 20130442.

15. Fricke-Zech S, Gruber RM, Dullin C, Zapf A, Kramer F-J, KubeinMeesenburg D, et al. Measurement of the midpalatal suture width: A comparison of flat-panel volume computed tomography to histomorphometric analysis in a porcine model. Angle Orthod 2012; 82(1): 145-50.

16. Revelo B, Fishman LS. Maturational evaluation of ossification of the midpalatal suture. Am J Orthod Dentofacial Orthop 1994; 105(3): 288-92.

17. Melsen B. Palatal growth studied on human autopsy material: a histologic microradiographic study. Am J Orthod 1975; 68(1): 42-54. 